\section{SP1-113 CLINICAL CORRELATION OF NON-ALCOHOLIC FATTY LIVER DISEASE IN A CHINESE TAXI DRIVERS POPULATION IN TAIWAN: EXPERIENCE AT A TEACHING HOSPITAL}

\author{
doi:10.1136/jech.2011.142976n.90
}

${ }^{1,2} \mathrm{~T}$ H Lin, ${ }^{*}{ }^{1,2} \mathrm{~T}$ H Tung, ${ }^{3,4} \mathrm{~W}$ H Chiu. ${ }^{1}$ Department of Public Health, College of Medicine, Fu-Jen Catholic University, Taipei, Taiwan; ${ }^{2}$ Cheng-Hsin General Hospital, Taipei, Taiwan; ${ }^{3}$ Department of Biomedical Imaging and Radiological Sciences, School of Biomedical Science and Engineering, National Yang-Ming University, Taipei, Taiwan; ${ }^{4}$ Central Clinic and Hospital, Taipei, Taiwan

Introduction To explore any gender-related differences in prevalence of and condition-associated factors related to non-alcoholic fatty liver disease (NAFLD) among Taiwanese taxi drivers in Taipei, Taiwan.

Methods We studied 1635 healthy taxi drivers (1541 males and 94 females) voluntarily admitted to physical check-up in 2006. Blood samples and ultrasound-proved fatty liver sonography results were collected.

Result The prevalence of NAFLD was $66.4 \%$ and revealed no statistically significant decrease with increasing age $(p=0.58)$. Males exhibited a greater prevalence of NAFLD than did females ( $67.5 \%$ vs $47.9 \%$, $\mathrm{p}<0.0001)$. Gender-related differences as regards associated factors were revealed. For males, hypertension, hyperuricemia, higher AST, higher ALT, hypertriglyceridemia, and higher fasting plasma glucose were significantly related to NAFLD but these were not so for females. Conclusion Several gender-related differences were noted pertaining to NAFLD among taxi drivers population.

\section{CUTTING EDGE METHODOLOGY}

\section{SP2-1 ROSE ANGINA QUESTIONNAIRE: ACCURACY FOR DIAGNOSING CORONARY HEART DISEASE IN BANGLADESH}

doi:10.1136/jech.2011.142976n.91

${ }^{1} \mathrm{M}$ A Rahman, ${ }^{1} \mathrm{~N}$ Spurrier, ${ }^{1} \mathrm{M}$ A Mahmood, ${ }^{2} \mathrm{M}$ Rahman, ${ }^{3} \mathrm{~S}$ R Choudhury, ${ }^{*}$ ${ }^{4} \mathrm{~S}$ Leeder. ${ }^{1}$ Discipline of Public Health, The University of Adelaide, Adelaide, South Australia, Australia; ${ }^{2}$ IEDCR (Institute of Epidemiology, Disease Control and Research), Dhaka, Bangladesh; ${ }^{3}$ NHFH\&RI (National Heart Foundation Hospital \& Research Institute, Dhaka, Bangladesh; ${ }^{4}$ The Menzies Centre for Health Policy, The University of Sydney, Sydney, New South Wales, Australia

Objective To determine accuracy of the Rose Angina Questionnaire (RAQ) for diagnosing coronary heart disease (CHD) among Bangladeshi adults, by comparing the classification based on the questionnaire with cardiologists' diagnosis.

Methods A case-control study of non-smoking Bangladeshi adults aged $40-75$ years, was conducted in 2010. Cases were incident cases of CHD from two cardiac hospitals if diagnosed as such by the cardiologists; controls were non-cardiac patients from cardiac outpatient departments. One control was matched to each case on age and gender. Full version of the original RAQ questionnaire was used for study participants.

Results The sample comprised 302 CHD cases and 302 controls (mean age $53 \pm 8.5$ years). RAQ detected 194 cases (32\%) and 409 controls (68\%) from either hospital. RAO categorised $17.5 \%$ of sample as having $\mathrm{CHD}$ who were considered not to have $\mathrm{CHD}$ by the cardiologists; RAQ categorised $34.5 \%$ of sample as not suffering from $\mathrm{CHD}$, who were diagnosed as having $\mathrm{CHD}$ by the cardiologists. Among $301 \mathrm{CHD}$ cases, 160 (53.2\%) were diagnosed as CHD by both hospital cardiologists and RAQ. Among 302 controls, 268 $(88.7 \%)$ were diagnosed as not having CHD by both hospital cardiologists and RAO. CHD patients are five times more likely to have RAQ positive result compared to controls.

Conclusion The RAQ had sensitivity of $53 \%$, specificity of $89 \%$ and likelihood ratio positive of 4.8 in diagnosing CHD among Bangladeshi adults compared with diagnosis by cardiologists. RAQ can be used as an alternate tool for diagnosing $\mathrm{CHD}$ at field sites where there are limited resources.

\section{SP2-2 COMMUNITY CONTROLS VS HOSPITAL CONTROLS: CHOICE FOR A CASE-CONTROL STUDY IN BANGLADESH}

doi:10.1136/jech.2011.142976n.92

${ }^{1} \mathrm{M}$ A Rahman, ${ }^{1} \mathrm{~N}$ Spurrier, ${ }^{1} \mathrm{M}$ A Mahmood, ${ }^{2} \mathrm{M}$ Rahman, ${ }^{3} \mathrm{~S}$ R Choudhury, ${ }^{4}$ S Leeder. 'Discipline of Public Health, The University of Adelaide, Adelaide, South Australia, Australia; ${ }^{2}$ IEDCR (Institute of Epidemiology, Disease Control and Research), Dhaka, Bangladesh; ${ }^{3}$ NHFH\&RI (National Heart Foundation Hospital \& Research Institute), Dhaka, Bangladesh; ${ }^{4}$ The Menzies Centre for Health Policy, The University of Sydney, Sydney, New South Wales, Australia

Objective To determine whether hospital controls could be used in case-control studies where resource constraints limit recruitment of community controls.

Methods Hospital controls and community controls were compared in terms of socio-demographic and risk factor variables in a study of smokeless tobacco (SLT) and coronary heart disease (CHD) in Bangladesh in 2010. Incident cases of CHD were selected from two cardiac hospitals. Hospital controls were selected from outpatient departments of the same hospitals. Community controls were selected from neighbourhoods matched to CHD cases. Four community controls and one hospital control were matched to each case on age and gender.

Results The study enrolled 302 cases, 1208 community controls and 302 hospital controls. There were no differences between hospital controls and community controls with respect to age, gender, marital status, occupation, economic status and risk factors for CHD. Hospital controls were more educated but less active physically than community controls. Current use of SLT was similar among community controls (33\%) and hospital controls (32\%), which was also not significant statistically (adjusted OR 0.81, 95\% CI 0.58 to 1.12, p>0.05). Current use of SLT was not associated with increased risk of $\mathrm{CHD}$ when data from community controls were used (adjusted OR 0.87, 95\% CI 0.63 to 1.19, p>0.05), nor when data from hospital controls were used (adjusted OR 1.00, 95\% CI 0.63 to $1.60, \mathrm{p}>0.05)$.

Conclusion For comparable future studies in situations of resource scarcity or difficult socio-political context, it is possible to enrol hospital controls with careful planning which are similar to potential community controls minimising bias

\section{SP2-3 GENOCIDE AND HEALTH: CHALLENGE FOR PUBLIC HEALTH AND EPIDEMIOLOGY}

doi:10.1136/jech.2011.142976n.93

$\mathrm{J}$ Lindert, ${ }^{*} 0$ von Ehrenstein. Protestant University, Ludwigsburg, Germany

Background Violence is the main cause of preventable death, worldwide. Research has shown that violence may develop in consecutive stages from the first stage (loss of opportunities for the persecuted group) to the last stages (loss of existence, loss of memory) in the aftermath. Each stage of violence can be measured with indicators that is, changes in language, devaluation of others, polarisation, preparation and denial. Further known indicators are the readiness to accept violence as a means for solving conflicts, inequality in distribution of resources and opportunities, level of discrimination and change in the legal context, percentage of weapons in a country, number of riots, discriminating of others in science and within the medical profession.

Objective We intend to provide the set of indicators and to introduce epidemiological tools for describing and understanding development of violence. 